\title{
Tamponada cardíaca por neumopericardio, a propósito de un caso
}

\author{
Cardiac tamponade due to pneumopericardium, about a case
}

Noel J. Díaz-Robles ${ }^{1 *}$, Mariano A. Chávez-Andino ${ }^{1}$, Verónica V. Gómez-Leiva ${ }^{2}$ y Enrique I. Orellana-Guevara ${ }^{1}$

${ }^{1}$ Servicio de Medicina Crítica de Adultos, Hospital Médico Quirúrgico del Instituto Salvadoreño del Seguro Social; ${ }^{2}$ Servicio de Medicina Nuclear de Adultos, Hospital de Oncología del Instituto Salvadoreño del Seguro Social. San Salvador, El Salvador

\section{Introducción}

El pericardio normalmente contiene $<50 \mathrm{ml}$ de líquido entre sus capas parietal y visceral, con una presión intrapericárdica que oscila entre $-5 \mathrm{y}+5 \mathrm{cmH}_{2} \mathrm{O}$. La cantidad y la velocidad de acumulación de un fluido determinan las repercusiones hemodinámicas ${ }^{1}$.

El pase de aire a la cavidad pericárdica se establece por causas traumáticas $(62 \%$, trauma penetrante o cerrado de tórax), no traumáticas $(29 \%$, ventilación mecánica con presión positiva, infecciosas por bacterias productoras de gas ${ }^{2}$, fístulas broncopleurales, fístulas pleuropericardicas) o iatrogénicas $(9 \%$, pericardiocentesis, pericardiotomía, toracocentesis, aspirado de médula ósea esternal, accesos venosos centrales, dispositivos de electroestimulación cardíaca) $)^{3,4}$.

En la literatura nacional solo se ha publicado un caso de neumopericardio asociado a trauma penetrante de tórax ${ }^{5}$.

Reportamos el caso de un neumopericardio a tensión postraumático asociado a neumomediastino y neumotórax cuya evolución fue favorable después de su drenaje.

\section{Descripción del caso}

Hombre de 36 años, sin antecedentes médicos, procedente y residente de San Salvador, quien es encontrado en la calle por agentes policiales posterior a sufrir un accidente de tránsito mientras conducía su motocicleta. Es llevado al hospital, donde le reciben con presión arterial de $80 / 50 \mathrm{mmHg}$, frecuencia cardíaca de 100 latidos/minuto, frecuencia respiratoria de 20 ciclos/minuto, saturación periferica de $\mathrm{O}_{2}$ del $88 \%$ al aire ambiente y Glasgow de 14 puntos. En cuanto a otros hallazgos clínicos, se evidencian pupilas isocóricas, nariz con restos hemáticos, enfisema subcutáneo, crepitación y desplazamiento de múltiples arcos costales en el hemitórax derecho. Se toman radiografías que evidencian fracturas costales derechas, neumotórax derecho, neumomediastino y neumopericardio (Fig. 1).

Se inicia la secuencia de inducción rápida, ventilación invasiva y tubo de toracostomía derecho. Se realiza lavado peritoneal diagnóstico, el cual es positivo, por lo que se lleva a cabo una laparotomía exploratoria que revela hemoperitoneo de $200 \mathrm{ml}$ y desgarro del mesenterio a $1.2 \mathrm{~cm}$ del ángulo de Treitz. Se realiza pericardiocentesis transdiafragmática drenando $50 \mathrm{ml}$ de sangre y no se evidencia salida de gas. Al mismo tiempo se efectúan toracotomía izquierda y pericardiotomía, drenando $20 \mathrm{ml}$ de hemopericardio. Se coloca un tubo de tórax izquierdo y finaliza la intervención.

Se complementan los estudios con tomografía computarizada cráneo-cérvico-tóraco-abdominal, que

Fecha de recepción: 02-09-2021

Fecha de aceptación: 08-12-2021

*Noel J. Díaz-Robles DOI: 10.24875/REIE.21000096

Disponible en internet: 07-03-2022

Rev Educ Investig Emer. 2022;4(Supl 1):51-54 www.medicinadeemergencias.com 
evidencia fracturas costales complejas desde el tercero hasta el séptimo arcos costales derechos, neumotórax laminar derecho, hemotórax laminar bilateral, neumopericardio y neumomediastino (Fig. 2). El ecocardiograma transtorácico, aun con mala ventana para su evaluación, muestra variación respiratoria del flujo de entrada transmitral > 20\% (Fig. 3).

La presunción diagnóstica de tamponada cardíaca por neumopericardio se basó en que el paciente presentaba inestabilidad hemodinámica y requerimiento de vasopresor (noradrenalina a $80 \mu \mathrm{g} / \mathrm{min}$ ). Se decide realizar ventana pericárdica, drenando $300 \mathrm{ml}$ de hemopericardio y aire a tensión, y se coloca un tubo de tórax del número 28.

El paciente evoluciona de manera favorable, con descenso del requerimiento vasopresor el mismo día del drenaje, y posteriormente pasa a fijación de fracturas costales hasta su egreso de la unidad de cuidados intensivos.

\section{Discusión}

La presencia de gas en el interior de la cavidad pericárdica está relacionado con múltiples causas, de las cuales la principal y más frecuente es el trauma. Cummings et al. ${ }^{3}$, en una revisión de 252 pacientes realizada en el año 1984, identificaron que el trauma se encontraba en un $62 \%$ de los casos, seguido de enfermedades de órganos vecinos (pulmón-pleura, esófago, estómago e hígado); en tres pacientes no pudo determinarse la causa.

Su presentación es poco frecuente, pero potencialmente mortal ${ }^{6}$. Se asocia con una mortalidad de hasta el $63.6 \%$, con repercusión grave cuando produce taponamiento cardíaco, el cual está presente en el $37 \%$ de los pacientes con diagnóstico de neumopericardio postraumático y en recién nacidos con ventilación con presión positiva ${ }^{6-8}$.

En la mayoría de los casos no causa compromiso hemodinámico, denominándose entonces neumopericardio que no provoca tensión (63\%), de evolución benigna y tratamiento conservador. Sin embargo, el $37 \%$ evoluciona a neumopericardio a tensión, implicando compromiso hemodinámico y tamponada cardíaca con necesidad de abordaje quirúrgico de drenaje (recomedacion IC) $)^{9}$.

El neumopericardio secundario a trauma cerrado de tórax se debe generalmente a tres mecanismos ${ }^{9}$ :

- Efecto de Macklin ${ }^{10}$, descrito por primera vez por el Dr. Charles Macklin, neumólogo canadiense que en el año 1939 realizó un estudio experimental con

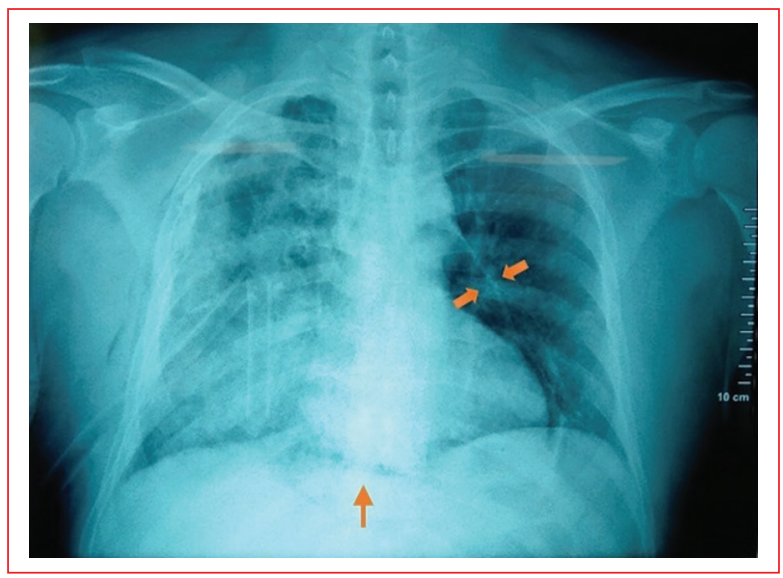

Figura 1. Radiografía de tórax. Imagen con aspecto de doble contorno (flechas gruesas) rodeando la silueta cardíaca. Signo del diafragma continuo (cúpula diafragmática visible, flechas finas). El tubo de tórax es recolocado.

animales (gatos y conejos) en el que demostró que la rotura alveolar daba paso a la disección de los espacios intersticiales pulmonares por el aire hasta alcanzar el mediastino y la cavidad pleural, y subsecuentemente la cavidad pericardica.

- Neumotórax con fístula pleuropericárdica.

- Comunicación o fístula traqueobroncopericárdica

En nuestro caso, sería difícil considerar que el neumopericardio de origen traumático asociado a neumotórax derecho y neumomediastino sea explicado por un mecanismo fisiopatológico de forma aislada. En su evolución, se realiza una broncoscopia que descarta la rotura del árbol traqueobronquial, por lo que la presencia de neumomediastino se explica por la rotura alveolar ante el incremento súbito de la presión positiva en la vía aérea durante el trauma, fenómeno explicado por Macklin ${ }^{10}$. Además, ante la presencia de neumotórax no podemos excluir una disrupción pleuropericárdica.

Ante un neumopericardio a tensión, el abordaje inicial es la descompresión pericárdica por medio de una pericardiocentesis realizada con una aguja para drenaje o mediante drenaje percutáneo de emergencia ${ }^{11}$ y 12 . Esta es una terapia temporal que sirve de puente hasta la realización de una ventana pericárdica permanente en la sala de operaciones, ya sea por abordaje subxifoideo abierto, toracotomía abierta o videotoracoscopía. Esta última tiene la ventaja de reducir los días con tubo de torax en pacientes traumatizados con fugas de aire persistentes ${ }^{13}$. 


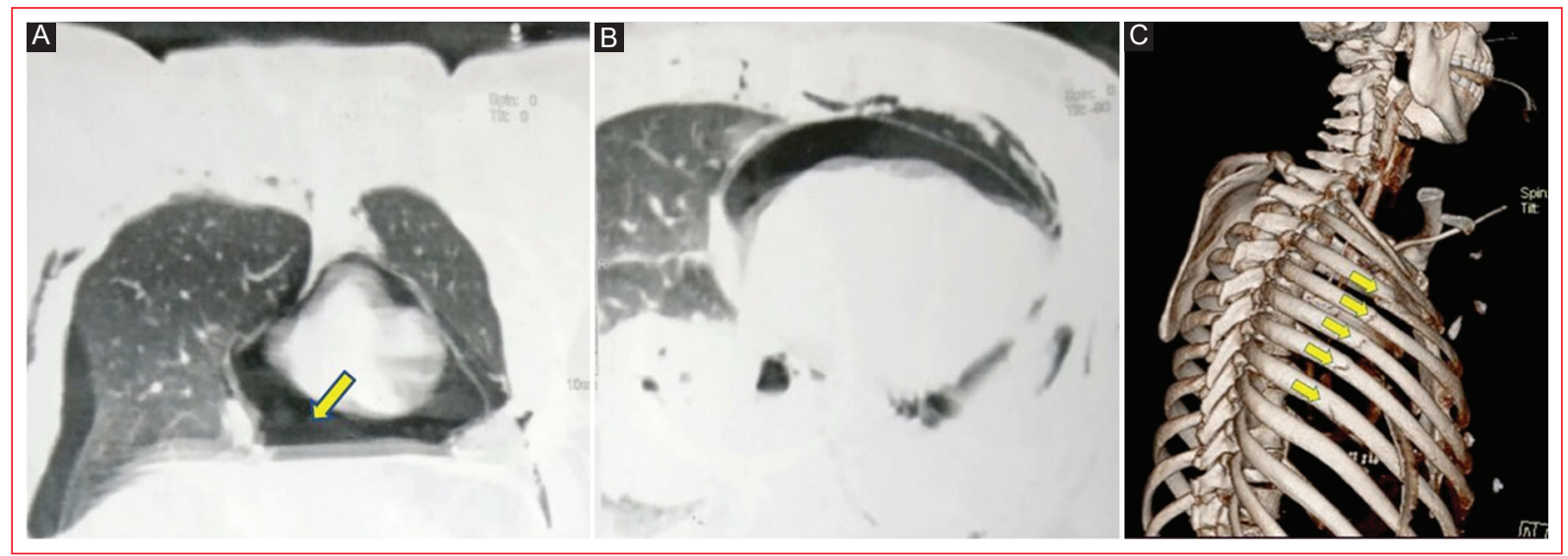

Figura 2. Tomografía computarizada de tórax. A y B: ventana pulmonar con neumotórax laminar derecho y neumopericardio a tensión (flecha). C: reconstrucción ósea con fracturas simples desplazadas del tercero al séptimo arcos costales derechos (flechas).

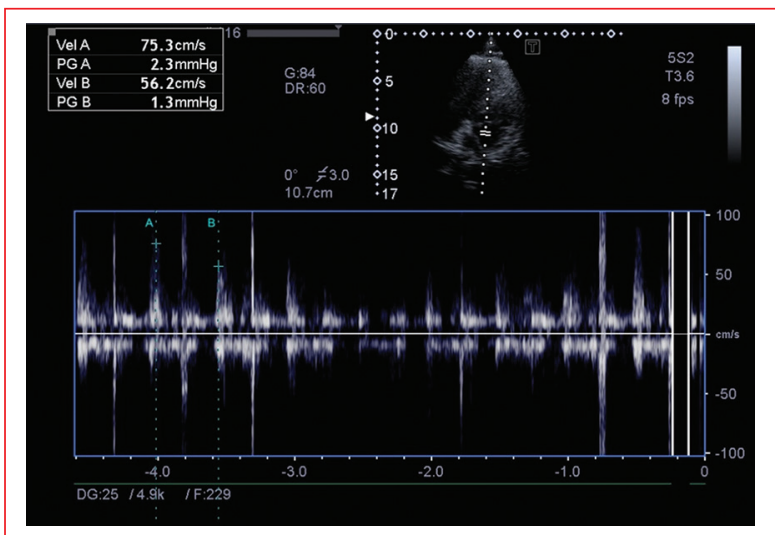

Figura 3. Ecocardiograma transtorácico. Variación respirofásica de flujo de entrada transmitral $>20 \%$ (mala ventana transtorácica).

Cuando existe comunicación entre el saco pericárdico y la cavidad pleural es preferible la colocación inicial del tubo de tórax; si dicha comunicación no existe, el drenaje pericárdico será de elección ${ }^{14}$.

El abordaje de nuestro paciente se inició con una ventana pericárdica transdiafragmática durante la laparotomía exploratoria, y la ausencia de salida de gas hizo decidir la no colocación de un drenaje pericárdico. Sin embargo, ante la persistencia del neumopericardio por imagen y el compromiso hemodinámico se realizó una segunda intervención con ventana pericárdica subxifoidea y colocacion de drenaje pericárdico con tubo y presión negativa.

\section{Conclusiones}

- El neumopericardio es poco frecuente y se requiere una alta sospecha clínica para poder diagnosticarlo. En el contexto de un trauma debe tomarse en cuenta como causa de deterioro hemodinámico después de descartar sangrado activo y neumotórax a tensión.

- El neumopericardio a tensión es una emergencia quirúrgica, ya que está asociado a tamponada y arresto cardíacos. El drenaje percutáneo debe realizarse inmediatamente y como terapia puente para la posterior colocación de un tubo pericárdico con presión negativa.

\section{Financiamiento}

Los autores declaran no haber recibido financiamiento para este estudio.

\section{Conflicto de intereses}

Los autores declaran no tener ningún conflicto de intereses.

\section{Responsabilidades éticas}

Protección de personas y animales. Los autores declaran que para esta investigación no se han realizado experimentos en seres humanos ni en animales. 
Confidencialidad de los datos. Los autores declaran que han seguido los protocolos de su centro de trabajo sobre la publicación de datos de pacientes.

Derecho a la privacidad y consentimiento informado. Los autores han obtenido el consentimiento informado de los pacientes y/o sujetos referidos en el artículo. Este documento obra en poder del autor de correspondencia.

\section{Bibliografía}

1. Hall J, Schmidt G, Kress J. Principles of critical care. $4^{\text {th }}$ ed. New York: McGraw-Hill; 2015.

2. Shivdasani B, Chhabria R, Mehta N. Pneumopericardium. Asian Cardiovasc Thorac Ann. 2017;25:476

3. Cummings R, Wesly R, Adams D, Lowe J. Pneumopericardium resulting in cardiac tamponade. Ann Thorac Surg. 1984;37:511-8.

4. Ouachaou J, Laaribi I, Mimouni H, Mellagui Y, Bkiyar H, Housni B. Post-traumatic compressive pneumopericardium with spontaneous ventilation: case report. Resp Med Case Rep. 2021;32:101354.

5. Merino Barrera SI, Moreno Martínez FL. Neumopericardio debido a herida por arma blanca. CorSalud. 2015;7:227-8.
6. Gutiérrez-Ospina A, Careaga-Reyna G, Lezama-Urtecho C, Quispe-Fernández L. Neumopericardio a tensión en trauma cerrado de tórax por efecto Macklin. Reporte de caso. Neumol Cir Torax. 2017;76:267-70.

7. Kallel N, Beloeil H, Geffroy A, Albaladejo P, Marty J. [Post-traumatic tension pneumothorax and pneumopericardium in spontaneous ventilation]. Ann Fr Anesth Reanim. 2004;23:364-6.

8. Capizzi PJ, Martin M, Bannon MP. Tension pneumopericardium following blunt injury. J Trauma. 1995;39:775-80.

9. Adler Y, Charron P, Imazio M, Badano L, Barón-Esquivias G, Bogaert J, et al. 2015 ESC Guidelines for the diagnosis and management of pericardial diseases: The Task Force for the Diagnosis and Management of Pericardial Diseases of the European Society of Cardiology (ESC). Endorsed by: The European Association for Cardio-Thoracic Surgery (EACTS). Eur Heart J. 2015;36:2921-64.

10. Macklin C. Transport of air along sheaths of pulmonic blood vessels from alveoli to mediastinum. Arch Intern Med. 1939;64:913.

11. Gould JC, Schurr MA. Tension pneumopericardium after blunt chest trauma. Ann Thorac Surg. 2001;72:1728-30.

12. Rolim Marques AF, Lopes LH, Martins M, Carmona CV, Fraga GP, Hirano ES. Tension pneumopericardium in blunt thoracic trauma. Int J Surg Case Rep. 2016;24:188-90.

13. Schermer CR, Matteson BD, Demarest GB $3^{\text {rd }}$, Albrecht RM, Davis VH A prospective evaluation of video-assisted thoracic surgery for persistent air leak due to trauma. Am J Surg. 1999;177:480-4.

14. Haddad R, Lima CE, Boasquevisque CH, Haddad GS, Ferreira TD. Pneumothorax and tension pneumopericardium following cardiothoracic surgery. J Bras Pneumol. 2006;32:84-7. 